\title{
Bounding Surface Approach to the Modeling of Anisotropic Fatigue Damage in Woven Fabric Composites
}

\author{
Chao Wen $^{1}$, Siamak Yazdani ${ }^{2}$, Yail J. Kim², Magdy Abdelrahman ${ }^{2}$ \\ ${ }^{1}$ Black \& Veatch Corporation, Overland Park, USA; ${ }^{2}$ North Dakota State University, Fargo, USA. \\ Email: wenc@bv.com, frank.yazdani@ndsu.edu,Jimmy.Kim@ndsu.edu, M.Abdelrahman@ndsu.edu
}

Received June $19^{\text {th }}, 2012$; revised July $14^{\text {th }}, 2012$; accepted July $23^{\text {rd }}, 2012$

\begin{abstract}
A general approach for the modeling of fatigue induced damage in woven fabric composites and under multi-axial stress state is outlined in this paper. Guided by isotropic hardening/softening theories of plasticity and damage mechanics, a generalized bounding surface approach is presented. It is argued that the limit surface is only a special case in such a formulation when the fatigue cycle is set to one and that under fatigue environment the limit surface contracts to a failure (residual strength) state based on the number of cycle, stress path, and stress magnitude. Within the formulation, specific kinetic relations for microcrack growth are postulated for woven fabric composites and a new direction function is specified to capture strength anisotropy of the material. Anisotropic stiffness degradations and inelastic strain propagation due to damage processes are also obtained utilizing damage mechanics formulation. The paper concludes with comparing theoretical predictions against experimental records showing a good agreement.
\end{abstract}

Keywords: Woven Composites; Fatigue; Damage; Anisotropic; Response Tensor

\section{Introduction}

A rather large number of scientific papers have been published on the modeling, simulation, and/or experimental investigation of composite materials under fatigue loading. The majority of the published works have addressed various topics associated with the uniaxial stress path loading. By comparison, the amount of work on the multiaxial modeling has been small. For one, the experimental testing under multiaxial stress state is difficult to conduct requiring special instrumentation and apparatus. This has lead to a small amount of experimental data to be available to develop and validate constitutive and failure models. However, the increasing use of woven fabric composites in structures subjected to complex loadings has required engineers to enhance the modeling and the predictive tools for a more reliable design. Compounding the difficulties is the complexity of microstructures of the woven composites itself and the presence of various defects and interfaces within the material.

There are three types of interfaces in woven composites [1-4]: resin rich area to longitudinal fiber group, resin rich area to transverse fiber group, and the interface between longitudinal fiber group and transverse fiber group. When loaded in the longitudinal direction, while the second kind of interface has little effects on the directions of crack propagation, the other two kinds of inter- faces tend to stop the development of cracks perpendicular to the direction of the load. Due to the strength and stiffness of the longitudinal fiber group, cracks propagating in the perpendicular direction stop at the longitudinal fiber group. The resultant stress concentration would then redirect cracks to the weak interface areas around the longitudinal fiber group and initiate breaking of interfaces between adjacent layers. After a number of the weak interfaces are broken down and resultant separate interface areas join together, delamination emerges. Under these complex phenomena, several different damage modes are present: micro-cracking, cracking, debonding, delamination, and fiber fracture [1-6]. Many researchers report that the fatigue process can be divided into three stages $[7,8]$. In the first stage, the main damage modes are matrix micro-cracking and cracking; the second stage is controlled by a combination of matrix cracking, interfacial cracking and delamination; while the fiber failure dominates the last stage.

Different approaches have been taken to address the presence of multitude of cracks as the main damage mode in fatigue process of composite materials. Broutman and Sahu [9] studied the progressive failure of the material by monitoring the crack density at the through thickness. Owen $[10,11]$ reported damage initiation at fiber-matrix interface due to debonding while, Mandell et $a l$. $[12,13]$ investigated fatigue damage propagation and 
failure modes of woven glass composites. Smith and Pasco $[1,6]$ investigated the behavior of glass reinforced composites under multiaxial state of stress in both monotonic and fatigue loading environment.

It is generally an accepted notion that modeling every crack or defect's evolution and growth is a formidable task, if not an impossible one. Therefore, many researchers have chosen to monitor changes in the material stiffness $[14,15]$ as an indirect but effective method to measure the internal changes and energy dissipation within the material due to damage.

Following this approach, Yoshioka and Seferis [16] presented a model to predict the fatigue process by observing modulus deterioration. Chou and Ko [17] proposed a model through the prediction of elastic stiffness based on lamination theory. Degrieck and Paepegem [18] have summarized the major fatigue models and life time prediction methodologies for reinforced polymer composites under fatigue loading. Recently, Wen and Yazdani [19] proposed a model to predict the fatigue process through the change of the fourth-order material compliance tensor based on a class of damage mechanics theories.

In this paper we propose a unified approach to the modeling of woven composites under quasi-static and fatigue loading utilizing the bounding surface approach. In fact it will be shown that the Limit (Strength) Surface "LS" is a special case when the fatigue cycle is set to one. This method has an intuitive advantage in that many in the mechanics community are familiar with the non-linear modeling of materials (plasticity and/or damage mechanics) and the extension to fatigue modeling would be regarded as natural. At the same time as will be shown many complex fatigue load paths can be modeled and addressed conveniently within the frame work proposed.

The concept of bounding surfaces can be explained as presented below. Consider a material element shown in Figure 1 where numbers "1" and "2" indicate orthogonal loading directions. Let the biaxial strength envelop (i.e., the Limit Surface representation in 2-D) of the material be represented by "LS" corresponding to the quasi-static loadings of the material point (Figure 2). The "LS" surface represents the limit (ultimate) strength of the material under a variety of loading paths in non-fatigue environment.

As understood in the fatigue loading, as the number of loading cycles increases, the ultimate strength of the material decreases due to the presence and activation of inherent and new flaws and damage in the material. In two dimensional representation scheme as we have adopted here, it is then plausible to consider that the Limit Surface, "LS", would collapse inward as represented by

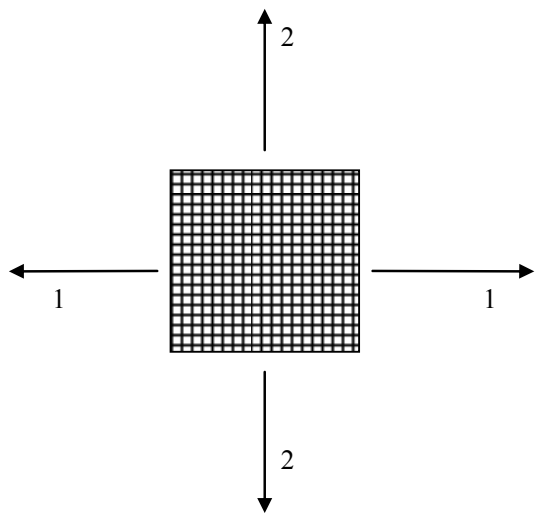

Figure 1. Material element with loading directions 1 and 2.

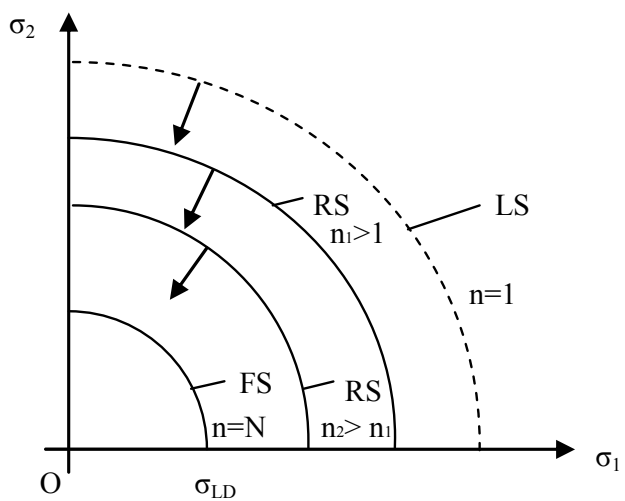

Figure 2. Schematic representation of boundary surfaces in two-dimensions.

"RS" family of curves identified as Residual Strength surfaces, utilizing the terminology used in fatigue literature. As the number of cycles increases the LS surface collapses further in as shown for $n_{2}>n_{1}$. At some point the failure state is reached where material fails due to the applied stress level at cycle "N". In fatigue literature, "N" is also referred to as life of the material. The task at hand is to develop a realistic and reasonable limit surface based on principles of mechanics, and to propose evolutionary equation that would provide the position of intermediate residual strength surfaces loading to the failure surface, FS, when $\mathrm{n}=\mathrm{N}$.

The model presented in the following sections is regarded as an extension to the work and Wen and Yazdani [19] where by utilizing a bounding surface theory and damage mechanics formulation a unified approach is presented for the fatigue modeling of woven fabric composites. A new direction function is also introduced to capture the strength anisotropy of the woven composite materials.

\section{Formulation}

In this paper it is assumed that the fatigue loading is of low frequency so that thermal effects could be ignored. 
With the further assumption of small deformation, the form of Gibbs Free Energy (GFE) is deduced from $[20,21]$ and shown as follows.

$$
G=\frac{1}{2} \sigma: C: \sigma+\sigma: \epsilon^{i}-A^{i}(k)
$$

where $\boldsymbol{C}$ represents the compliance tensor, $\boldsymbol{\varepsilon}^{i}$ designates the inelastic strain tensor, $\sigma$ is the applied stress tensor, $A^{i}(k)$ is a scalar function, and $\mathrm{k}$ is the cumulative fatigue damage parameter. The symbol ":" denotes the tensor contraction operation. For small deformations as assumed, one can decompose the current compliance tensor into an initial undamaged component plus added flexibility caused by damage during fatigue loadings as [20-24]:

$$
\boldsymbol{C}(k)=\boldsymbol{C}^{\mathbf{0}}+\boldsymbol{C}^{c}(k)
$$

where, $\boldsymbol{C}^{0}$ is the initial undamaged fourth-order compliance tensor and $\boldsymbol{C}^{c}(k)$ denotes the added flexibility tensor due to damage. Also, the changes in the fourthorder compliance tensor and the inelastic strain tensor are regarded as fluxes in the thermodynamic state sense and are expressed below with respect to a set of response tensors $\boldsymbol{R}$ and $\boldsymbol{M}$ as:

$$
\dot{\boldsymbol{C}}^{c}=\dot{k} \boldsymbol{R} \text { and } \dot{\boldsymbol{\varepsilon}}^{i}=\dot{k} \boldsymbol{M}
$$

The response tensors determine the directions of the fatigue damage and the inelastic deformation processes. Following the standard thermodynamics arguments and assuming that the unloading is an elastic process, the onset of damage is determined by defining a potential function $\psi(\sigma, k)$ that is derived by combining Equations (1)-(3) so that [20,21]

$$
\psi(\boldsymbol{\sigma}, k)=\frac{1}{2} \boldsymbol{\sigma}: \boldsymbol{R}: \boldsymbol{\sigma}+\boldsymbol{\sigma}: \boldsymbol{M}-\frac{1}{2} t^{2}(\boldsymbol{\sigma}, k)=0
$$

where $t(\sigma, k)$ is interpreted as the damage function given below as

$$
t^{2}(\sigma, k)=2\left[\frac{\partial A^{i}}{\partial k}+g^{2}(\sigma, k)\right]
$$

for some scalar-valued function $g^{2}(\sigma, k)$. We note that as long the function " $t$ " could be obtained experimentally, the identification of the components shown on the right hand side of the Equation (5) is not necessary.

To progress further, specific forms of the response tensors $\boldsymbol{R}$ and $\boldsymbol{M}$ must be provided. Guided by the experimental data from literature [1], the following response tensors are postulated for $\boldsymbol{R}$ and $\boldsymbol{M}$ :

$$
\begin{gathered}
\boldsymbol{R}=\frac{\boldsymbol{\sigma} \otimes \boldsymbol{\sigma}}{\boldsymbol{\sigma}: \boldsymbol{\sigma}}+\alpha(I-\boldsymbol{i} \otimes \boldsymbol{i}) \\
\boldsymbol{M}=\beta \boldsymbol{\sigma}
\end{gathered}
$$

where the symbol " $\otimes$ " signifies the tensor product op- eration, $\boldsymbol{I}$ represents the fourth-order identity tensor, $\boldsymbol{i}$ represents the second-order identity tensor, and $\alpha$ and $\beta$ are material parameters.

The response tensor $\boldsymbol{R}$ is composed of two parts as follows.

$$
\begin{gathered}
\boldsymbol{R}_{\boldsymbol{I}}=\frac{\boldsymbol{\sigma} \otimes \boldsymbol{\sigma}}{\sigma: \sigma} \\
\boldsymbol{R}_{\boldsymbol{I I}}=\alpha(\boldsymbol{I}-\boldsymbol{i} \otimes \boldsymbol{i})
\end{gathered}
$$

The first part, $\boldsymbol{R}_{\boldsymbol{I}}$, indicates that damage occurs in the loading directions. This is in concurrence with observed experimental data [1]. However with $\boldsymbol{R}_{\boldsymbol{I}}$ alone, the limit surface that is predicted by the model cannot match the experimental data as shown in Figure 3. Also, with $\boldsymbol{R}_{\boldsymbol{I}}$ alone any change in the Poisson's ratio could not be predicted by the proposed theory. Thus, the second part, $\boldsymbol{R}_{\boldsymbol{I I}}$, is included. With an experimentally determined value of parameter $\alpha$, the limit surface prediction is shown as the solid curve in Figure 3. The role of $\boldsymbol{R}_{I I}$ is thus two fold. One, the form enables the model to predict enhancement in strength under proportional loading, and two enables the model to address changes in the apparent poison's ratio.

The damage function, $t(\sigma, k)$, is further represented as the product of two functions $L(\sigma)$ and $q(k)$ such that

$$
t(\sigma, k)=L(\sigma) q(k)
$$

where $L(\sigma)$ and $q(k)$ are interpreted as the strength and the shape functions of the damage function, $\mathrm{t}$, with a condition that $q_{\max }(k)=1$, that is the maximum value of the function $q(k)$ is set to be one. Considering a class of woven composites $(0-90)$, we identify a strength tensor, $\boldsymbol{S}$, as,

$$
\boldsymbol{S}=F_{t 1} \boldsymbol{q}_{1} \otimes \boldsymbol{q}_{1}+F_{t 2} \boldsymbol{q}_{2} \otimes \boldsymbol{q}_{2}
$$

where $F_{t 1}$ and $F_{t 2}$ are scalar parameters, and $\boldsymbol{q}_{\mathbf{1}}$ and $\boldsymbol{q}_{2}$ are Eigen vectors of fiber directions, respectively. It will be shown below that $F_{t 1}$ and $F_{t 2}$ are related to the material strengths $f_{t 1}$ and $f_{t 2}$ in direction " 1 " and " 2 ", respectively. With these backgrounds in place, a particular form for the strength function $L(\sigma)$ of Equation (10) is proposed as

$$
L(\sigma)=\frac{\sigma: S}{\operatorname{Tr}(\sigma)}
$$

By substituting Equations (6), (7), (10), and (12) into Equation (4), the damage function is re-written as

$$
\begin{aligned}
\psi(\sigma, k)= & \frac{1}{2}(1+\alpha+2 \beta) \sigma: \sigma-\frac{1}{2} \alpha[\operatorname{Tr}(\sigma)]^{2} \\
& -\frac{1}{2}\left[\frac{\boldsymbol{\sigma}: \boldsymbol{S}}{\operatorname{Tr}(\boldsymbol{\sigma})} q(k)\right]^{2}=0
\end{aligned}
$$




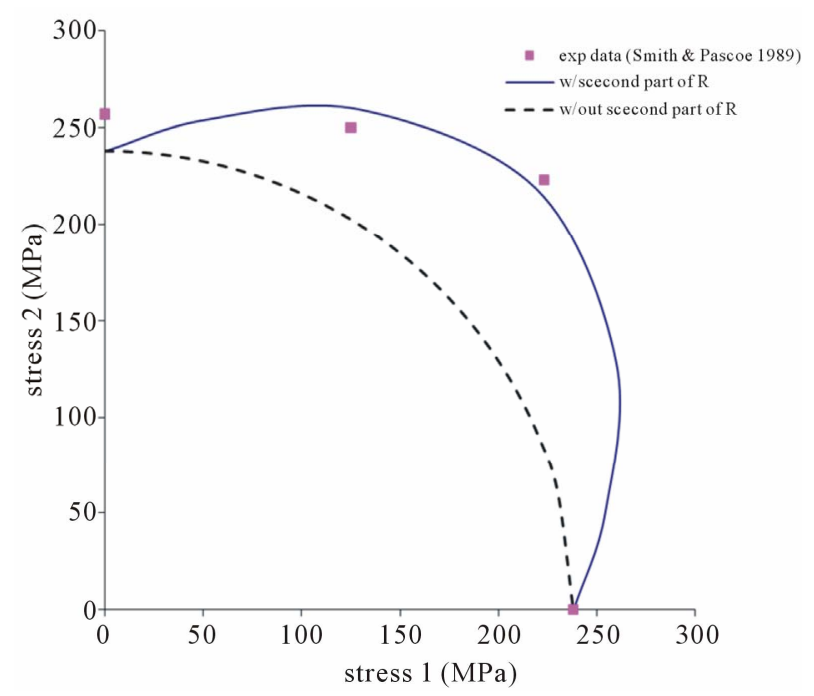

Figure 3. Effect of the two parts of response tensor $\mathbf{R}$. Experimental data are of the biaxial ultimate strengths of specimen [1].

To obtain the forms of scalar parameters $F_{t 1}$ and $F_{t 2}$, two uniaxial loading paths in fiber direction " 1 " and " 2 " at the limit state are considered, respectively. Since at the limit state the function $q(k)=1$, in direction " 1 " Equation (13) is simplified to be as

$$
F_{t 1}^{2}=f_{t 1}^{2}(1+2 \beta)
$$

where $f_{t 1}$ is the tensile strength in direction " 1 ". Similarly, in direction " 2 ", designating $f_{t 2}$ as the tensile strength in direction " 2 ", Equation (13) is simplified to be as:

$$
F_{t 2}^{2}=f_{t 2}^{2}(1+2 \beta)
$$

It is observed that if $f_{t 1}$ and $f_{t 2}$ are the same, the model will predict strength isotropy; while with $f_{t 1}$ and $f_{t 2}$ being unequal, the model will predict strength anisotropy as one expects in most composites.

An example is provided here to illustrate the capability of the model to predict strength isotropy and anisotropy. The predicted limit surfaces of two materials are shown in Figure 4. The dashed curve represents a material with strength anisotropic with strength $f_{t 1}=80 \mathrm{MPa}$ in direction " 1 " and $f_{t 2}=50 \mathrm{MPa}$ in direction " 2 ." The solid curve represent a material with a strength isotropy with strength $f_{t 1}=f_{t 2}=80 \mathrm{MPa}$ in both directions.

\section{Fatigue}

As the number of loading cycles starts to increase, the strength of the material is affected and reduced. The limit surface representing the foci of all strength points associated with $n=1$ is therefore affected and should be modeled to soften to failure surface. To achieve this, the strength function $L(\sigma)$ is modified to predict lower

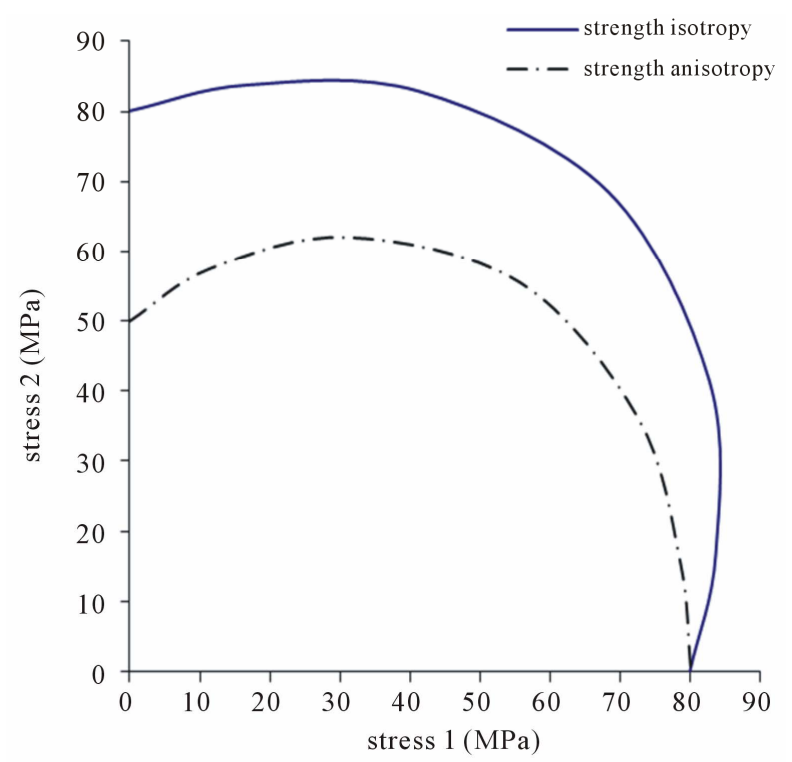

Figure 4. Schematic illustration of model predicted limit surface for strength isotropic and anisotropic materials.

limit strength of the material with increasing number of cycles. Therefore, a new strength function, $L(\sigma, n)$ is proposed as

$$
L(\boldsymbol{\sigma}, n)=\frac{\boldsymbol{\sigma}: \boldsymbol{S}}{\operatorname{Tr}(\boldsymbol{\sigma})} F(n)
$$

where $F(n)$ acts as a the softening function.

Incorporating the damage softening function back into the general formulation yields

$$
\begin{aligned}
\psi(\boldsymbol{\sigma}, k)= & \frac{1}{2}(1+\alpha+2 \beta) \boldsymbol{\sigma}: \boldsymbol{\sigma}-\frac{1}{2} \alpha(\operatorname{Tr}(\boldsymbol{\sigma}))^{2} \\
& -\frac{1}{2}\left[\frac{\boldsymbol{\sigma}: \boldsymbol{S}}{\operatorname{Tr}(\boldsymbol{\sigma})} F(n) q(k)\right]^{2}=0
\end{aligned}
$$

To interpret the softening function $F(n)$ we consider a uniaxial fatigue loading path in the fiber direction " 1 " with $q_{\max }=1$ as:

$$
\begin{aligned}
\psi(\sigma, k)= & \frac{1}{2}(1+\alpha+2 \beta) \sigma_{1}^{2}-\frac{1}{2} \alpha\left(\sigma_{1}\right)^{2} \\
& -\frac{1}{2}\left[F_{t 1} F(n)\right]^{2}=0
\end{aligned}
$$

With the previously obtained result that $F_{t 1}{ }^{2}=f_{t 1}{ }^{2}(1+2 \beta)$, we obtains the expression for $F(n)$ as

$$
F(n)=\frac{\sigma_{1}}{f_{t 1}}
$$

The relation (19) represents the ratio of the residual strength over the ultimate quasi-static strength. This is also referred to the classical S-N curve in fatigue literature terminologies. To determine a proper form for 
$F(n)$, we therefore refer to the experimental S-N curve for uniaxial tension in the literature. The two most fundamental classical S-N curves are the power function and logarithm function. By comparison with experimental work [1], the power function is used as follows. Let

$$
F(n)=n^{A}
$$

where $n$ is the number of cyclic loading, and $A$ is a material parameter.

For the experimental work [1] that is used here, the model predictions are shown in Figures 5-7 for various stress ratios.

To obtain the constant material parameter " $A$ ", we utilize Equations (14) and (20) to get

$$
A=\ln \left(\frac{\sigma_{1}}{f_{t 1}}\right) / \ln (n)
$$

Finally, the rate of the damage parameter, $k$, must be obtained and specified consistent with the constitutive relations used and the strength degradation forms proposed due to fatigue cycles. For the simple constitutive relation of the form shown as $\varepsilon=C(k): \sigma+\varepsilon i$, the rate of damage parameter, $d k / d n$, for the uniaxial path can be shown to be as

$$
\frac{\mathrm{d} k}{\mathrm{~d} n}=\frac{1}{E_{0}(1+\beta)}(-A)\left(n^{-A-1}\right)
$$

where $E_{0}$ is the initial Young's modulus in the absence of any damage.

\section{Numerical Simulation}

In this section, the predictions of the proposed model are compared with the experimental data [1]. Smith \& Pascoe [1] used a biaxial hydraulic servo-controlled rig developed at the Cambridge University Engineering Department. Nine biaxial and three uniaxial stress states were tested. All tests were load control. Fatigue test frequencies were generally kept in the range $0.1 \mathrm{~Hz}-0.6 \mathrm{~Hz}$ to prevent excessive cyclic induced heating. The specimens were cruciform for biaxial tests and parallel-sided for uniaxial tests. All specimens were from one batch of laminate which was laid up from reinforcement of glass fiber woven roving $(0-90)$ and isophthalic polyester resin. Each laminate contains 13 laminas. Warp and weft fibers of different lamina are aligned in the same directions, respectively.

Three material parameters, $A, \alpha$, and $\beta$ are used in the model. To determine the parameters, the following tests can be used. With one uniaxial fatigue test and knowing the uniaxial strength of the material, the residual strength, $\sigma_{1}$, and cyclic number, n, can be obtained. The constant $A$ can then be determined by Equation (21). The parameter $\beta$ is a kinematic parameter and is identified by measuring

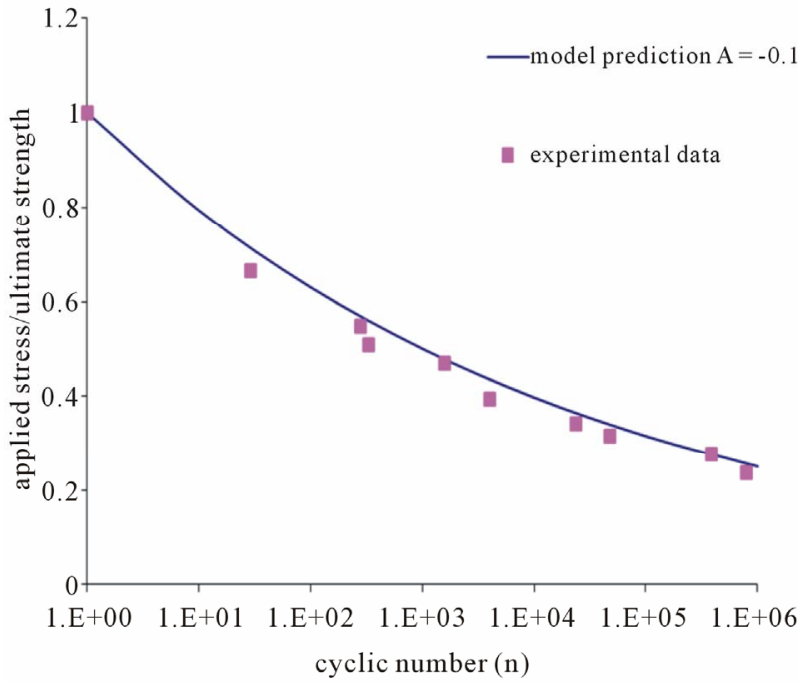

Figure 5. Comparison between softening function and experimental data [1] with stress ratio 1:0.

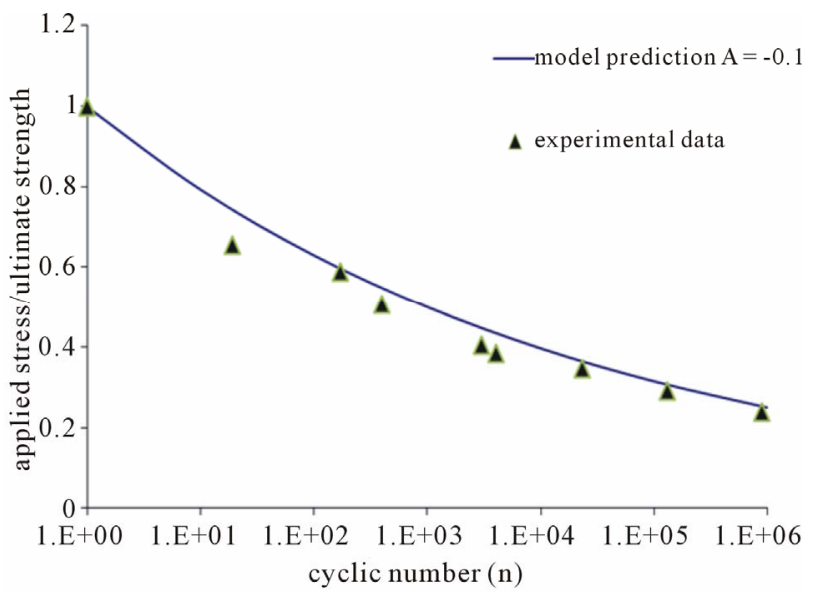

Figure 6. Comparison between softening function and experimental data [1] with stress ratio 1:0.5.

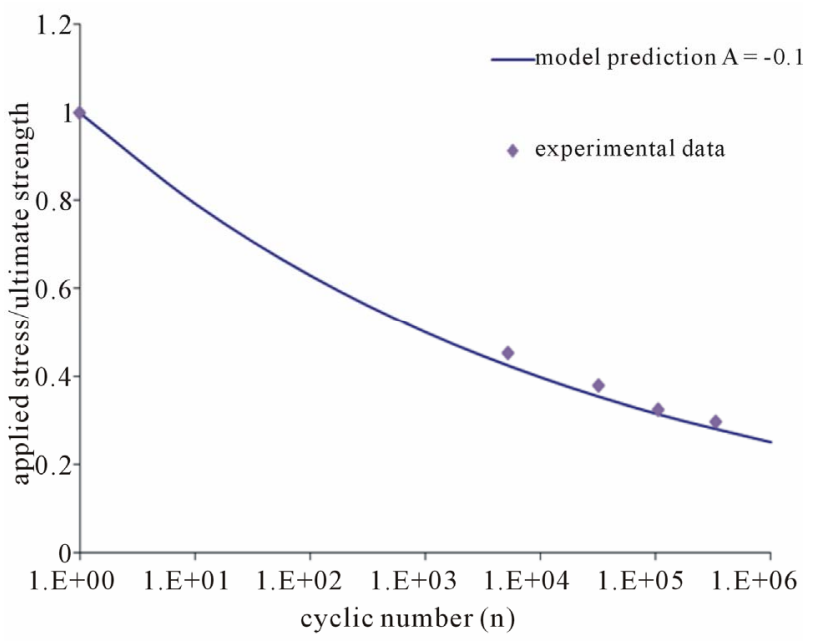

Figure 7. Comparison between softening function and experimental data [1] with stress ratio 1:1. 
the inelastic deformation after unloading. With one biaxial quasi-static test and parameter $\beta, \alpha$ can be determined by Equation (13).

Figure 8 shows the prediction results of biaxial limit surface and residual strength surface against the experimental work [1], for the monotonic loading when $n=1$ and the fatigue loading when $n=105$ cycles. The theoretical results for predictions are good considering the simplicity of the forms that were used. The following material parameters were used: $\alpha=0.46, \beta=0.1, A=$ -0.1 .

Figure 9 shows the comparison of the model prediction of the increment of compliance with the experimental data [1]. The experimental data are of an equal biaxial fatigue test. The values of parameters $\alpha, \beta$, and $A$ are the same as those of Figure 8. Lastly, the predicted stress-strain relations are shown on Figures 10-12 where the strength and ductility reductions are demonstrated due to effect of fatigue loading. Figure 10 shows the predicted stress-strain relations of uniaxial monotonic and fatigue loadings; Figure 11 shows those of monotonic and fatigue loadings with stress ratio 1:0.5; Figure 12 shows those of monotonic and fatigue loadings with stress ratio 1:1. The experimental data are from the work [1].

\section{Conclusion}

An anisotropic damage model is established to predict the fatigue behavior of woven composite materials under low frequency fatigue loading for multi-axial stress states. A class of damage mechanics is utilized recognizing that cracking is the main type of irreversible process and damage in the material that also dominates most of the

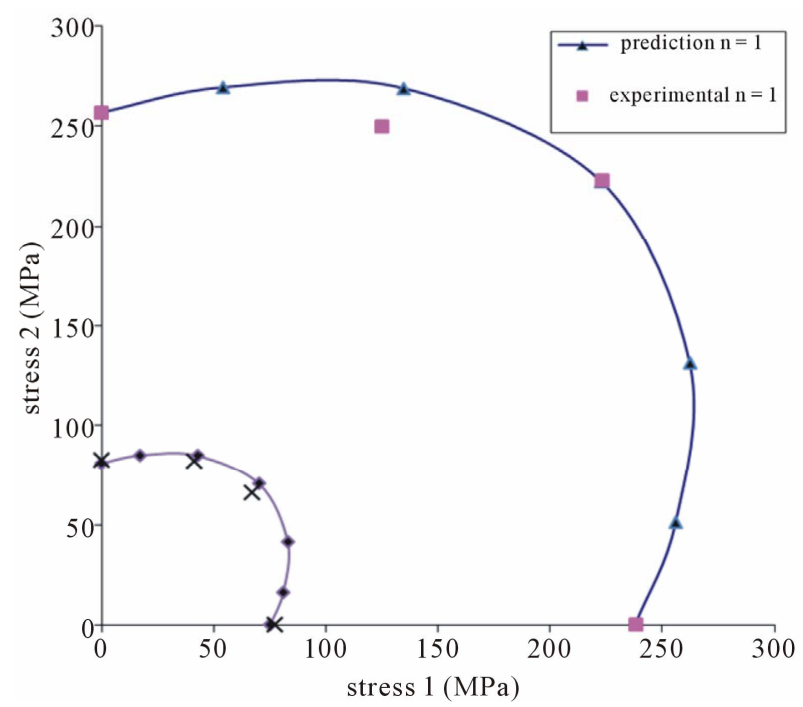

Figure 8. Comparison between experimental data [1] and theory predictions of limit surface and residual strength surface of 105 loading cycles.

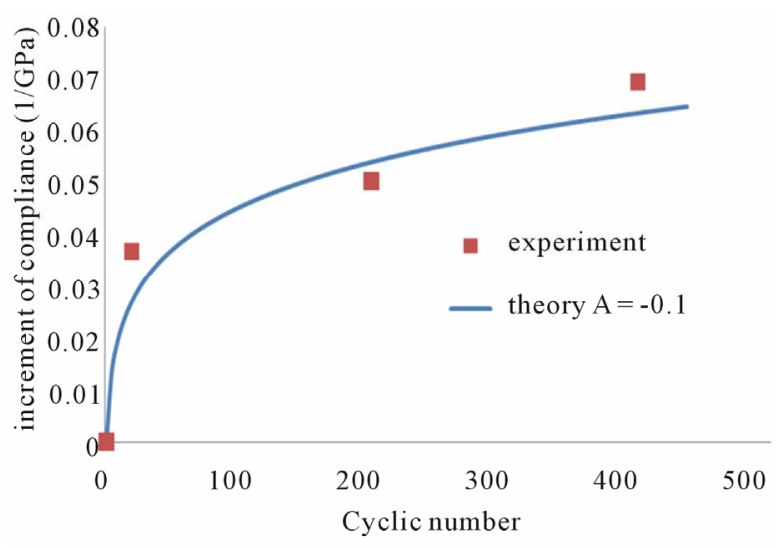

Figure 9. Comparison of increment of compliance of equal biaxial fatigue between experimental data [1] and model prediction.

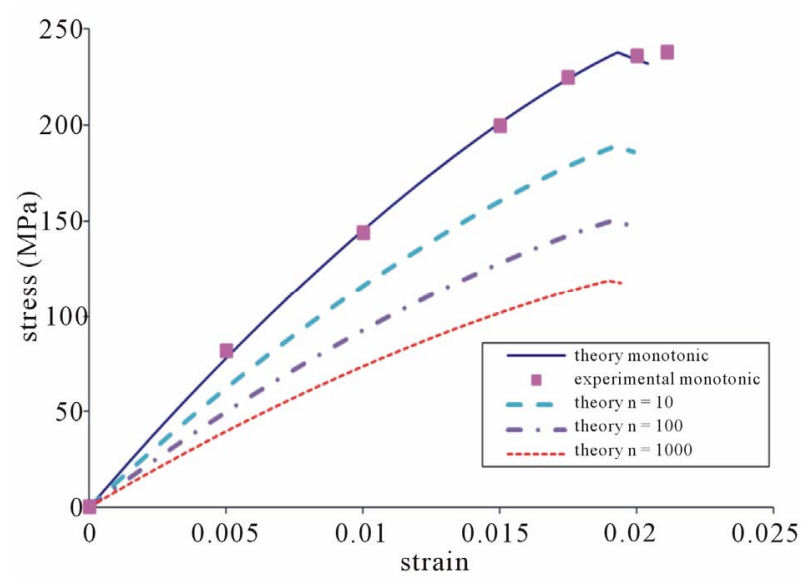

Figure 10. Predictions of the stress strain relationship of uniaxial monotonic failure loading and uniaxial fatigue loadings. The experimental data are from the work of Smith \& Pascoe [1].

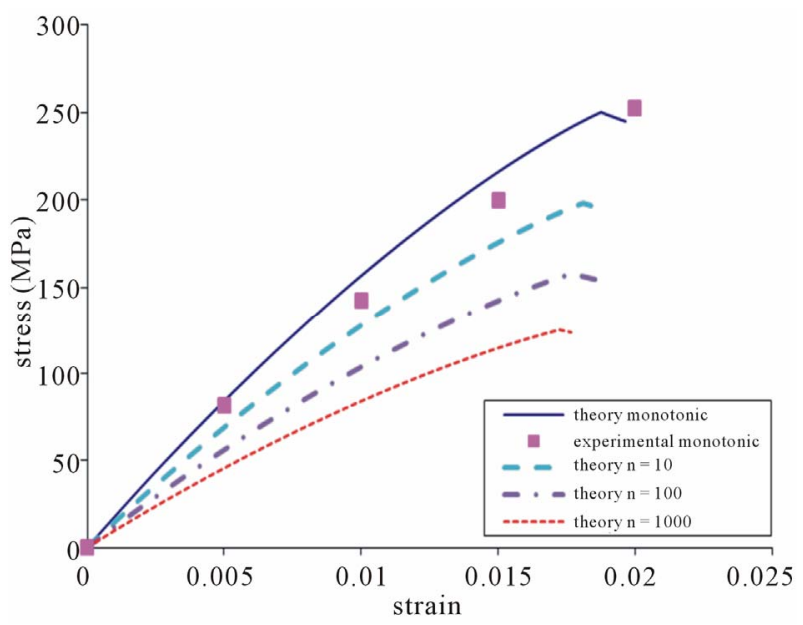

Figure 11. Predictions of the stress strain relationship of monotonic failure loading and fatigue loadings with stress ratio 1:0.5. The experimental data are from the work of Smith \& Pascoe [1]. 


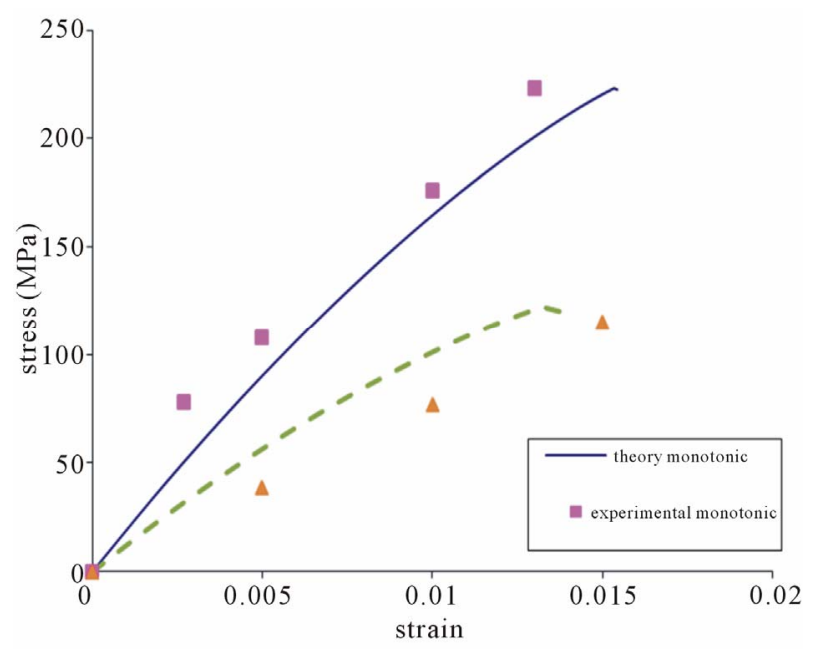

Figure 12. Predictions of the stress strain relationship of monotonic failure loading and fatigue loadings with stress ratio 1:1. The experimental data are from the work of Smith \& Pascoe [1].

fatigue life. In this work a bounding surface theory is presented to predict the fatigue behavior of the woven material under biaxial loadings. The limit strength state expressed as a potential damage function is let to soften (shrink) based on an appropriate rate of a damage variable. The changes of the material properties and the inelastic deformation are also addressed by means of response tensors. The forms of response tensors allow the formulation to predict induced anisotropy due to cracking. Strength anisotropy is also studied and addressed. By comparison with experimental data, the model shows good capability to describe the essential properties of woven composite materials under biaxial fatigue loading.

\section{REFERENCES}

[1] E. W. Smith and K. J. Pascoe, "Biaxial Fatigue of a Glass-Fiber Reinforced Composite. I. Fatigue and Fracture Behavior," Mechanical Engineering Publications, Biaxial and Multiaxial Fatigue, 1989, pp. 367-396.

[2] A. J. Russell, "Assessing the Long Term Durability of Glass Fibre Composites," The 7th International Conference on Marine Application of Composite Materials, 1998, pp. R1-R13.

[3] Z. Khan, et al., "Fatigue Damage Characterization in Plain Weave Carbon-Carbon Fabric Reinforced Plastic Composites," Journal of Reinforced Plastics and Composite, Vol. 17, No. 15, 1998, pp. 1320-1337.

[4] W. W. Stinchcomb and K. L. Reifsnider, "Fatigue Damage Mechanisms in Composite Materials: A Review," Fatigue Mechanisms, 1979, pp. 762-787.

[5] H. A. Whitworth, "A Stiffness Degradation Model for Composite Laminates under Fatigue Loading," Composite Structures, Vol. 40, No. 2, 1998, pp. 95-101. doi:10.1016/S0263-8223(97)00142-6
[6] E. W. Smith and K. J. Pascoe, "Biaxial Fatigue of a GlassFiber Reinforced Composite, II. Failure Criteria for Fatigue and Fracture," Mechanical Engineering Publications, Biaxial and Multiaxial Fatigue, 1989, pp. 397-421.

[7] U. Hansen, "Damage Development in Woven Fabric Composites during Tension-Tension Fatigue," Journal of Composite Materials, Vol. 33, No. 7, 1999, pp. 614-639. doi:10.1177/002199839903300702

[8] V. Natarajan, H. V. S. Gangarao and V. Shekar, "Fatigue Response of Fabric-Reinforced Polymeric Composites," Journal of Composite Materials, Vol. 39, No. 17, 2005, pp. 1541-1559. doi:10.1177/0021998305051084

[9] L. J. Boutman and S. Sahu, "Mechanical Properties of Particulate Composites," Polymer Engineering and Science, Vol. 12, 1972, pp. 91-100. doi:10.1002/pen.760120204

[10] M. J. Owen, "Fatigue Processes in Fibre Reinforced Plastics," Philosophical Transactions, Series A, Vol. 294, No. 1411, 1980, pp. 535-543. doi:10.1098/rsta.1980.0062

[11] M. J. Owen, "Fatigue Testing of Fiber Reinforced Plastics," Composites, Vol. 1, No. 6, 1970, pp. 346-355. doi:10.1016/0010-4361(70)90233-8

[12] J. Mandell, D. Cairns, D. Samborsky, R. Morehead and D. Haugen, "Prediction of Delamination in Wind Turbine Blade Structural Details," The 41st AIAA Aerospace Sciences Meeting and Exhibit, Reno, 2003, pp. 202-213.

[13] J. F. Mandell, D. H. Grande, T. H. Tsiang and F. J. McGarry, "Modified Mirco-Debonding Test for Direct in Situ Fiber/Matrix Bond Strength Determination in Fiber Composites," Composite Materials: Testing and Design, the 7th Conference, Philadelphia, 2-4 April 1984, pp. 87108.

[14] A. L. Highsmith and K. L. Reifsnider, "Stiffness-Reduction Mechanisms in Composite Laminates," Damage in Composite Materials: Basic Mechanisms, Accumulation, Tolerance, and Characterization, American Society for Testing and Materials, Philadelphia, 1982, pp. 103-117.

[15] J. Degrieck and W. Van Paepegem, "Fatigue Damage Modeling of Fiber-Reinforced Composite Materials: Review," Applied Mechanics Review (USA), Vol. 54, No. 4 2001, pp. 279-300. doi:10.1115/1.1381395

[16] K. Yoshioka and J. C. Seferis, "Modeling of Tensile Fatigue Damage in Resin Transfer Molded Woven Carbon Fabric Composites," Composites: Part A, Vol. 33, No. 11, 2002, pp. 1593-1601.

[17] T. W. Chou and F. K. Ko, "Textile Structural Composites," Elsevier Science Publishers, Amsterdam, 1989.

[18] J. Degrieck and W. V. Paepegem, "Coupled Residual Stiffness and Strength Model for Fatigue of Fiber-Reinforced Composite Materials," Composite Science and Technology, Vol. 62, No. 5, 2002, pp. 687-696. doi:10.1016/S0266-3538(01)00226-3

[19] C. Wen and S. Yazdani, "Anisotropic Damage Model for Woven Fabric Composites during Tension-Tension Fatigue," Composite Structures, Vol. 82, No. 1, 2008, pp. 127 131. doi:10.1016/j.compstruct.2007.01.003

[20] S. Yazdani and H. L. Schreyer, "Combined Plasticity and 
Damage Mechanics Model for Plain Concrete," Journal of Engineering Mechanics, Vol. 116, No. 7, 1990, pp. 14351450. doi:10.1061/(ASCE)0733-9399(1990)116:7(1435)

[21] S. Yazdani and S. A. Karnawat, "A Constitutive Theory for Brittle Solids with Application to Concrete," International Journal of Damage Mechanics, Vol. 21, No. 8, 1996, pp. 93-110. doi:10.1177/105678959600500105

[22] B. Budiansky and R. J. O'Connell, "Elastic Moduli of a Cracked Solid," International Journal of Solids and Struc- tures, Vol. 12, No. 2, 1976, pp. 81-97. doi:10.1016/0020-7683(76)90044-5

[23] H. Horri and S. Nemat-Nasser, "Overall Moduli of Solids with Microcracks: Load-Induced Anisotropy," Journal of the Mechanics and Physics of Solids, Vol. 31, No. 2, 1983, pp. 155-171. doi:10.1016/0022-5096(83)90048-0

[24] M. Ortiz, "A Constitutive Theory for the Inelastic Behavior of Concrete," Mechanics of Materials, Vol. 4, No. 1, 1985, pp. 67-93. doi:10.1016/0167-6636(85)90007-9 